\title{
Current research progress in the role of reactive oxygen species in esophageal adenocarcinoma
}

\author{
Youzhen Hu${ }^{1 \#}$, Xiaojun Ye ${ }^{2 \#}$, Ruihua Wang ${ }^{1}$, Karen Poon ${ }^{2} \wedge$ \\ ${ }^{1}$ Department of Gastroenterology, Shenzhen Hospital, Southern Medical University, Shenzhen, China; ${ }^{2}$ Program of Food Science and Technology, \\ Division of Science and Technology, BNU-HKBU United International College, Zhuhai, Guangdong, China \\ Contributions: (I) Conception and design: K Poon, R Wang; (II) Administrative support: Y Hu, X Ye; (III) Provision of study materials or patients: \\ Y Hu, X Ye; (IV) Collection and assembly of data: Y Hu, X Ye; (V) Data analysis and interpretation: Y Hu, X Ye; (VI) Manuscript writing: All authors; \\ (VII) Final approval of manuscript: All authors. \\ "These authors contributed equally to this work. \\ Correspondence to: Ruihua Wang, MD. Department of Gastroenterology, Shenzhen Hospital, Southern Medical University, Shenzhen 518100, China. \\ Email: ruihuawang@vip.163.com; Karen Poon, PhD. Program of Food Science and Technology, Division of Science and Technology, BNU-HKBU \\ United International College, 2000 Jintong Road, Tangjiawan, Zhuhai 519085, China. Email: karenpoon@uic.edu.cn.
}

\begin{abstract}
In the past few decades, the incidence of esophageal adenocarcinoma has increased by six-fold in western countries, as the proton pump inhibitor targeting the gastric acid reflux has failed to control the disease. It is currently suggested that deoxycholic acid reflux leads to esophageal adenocarcinoma. As an inflammation-related cancer, the formation and progression of esophageal adenocarcinoma are closely related to the concentration of reactive oxygen species (ROS). Meanwhile, the critical developmental stage of esophageal adenocarcinoma involves characteristic pathological changes in which the distal esophageal squamous epithelial cells are replaced by intestinal columnar epithelial cells, suggesting the involvement of cancer stem cells. Thus, esophageal adenocarcinoma is a good model to study the interplay between ROS and stem cells in cancer. Until now, some important questions related to ROS in esophageal adenocarcinoma remain unanswered. For example, the molecular mechanism by which deoxycholic acid induces malignant transformation in esophageal adenocarcinoma remains unclear. In addition, whether ROS are involved in the induction of cancer stem cell formation by chemotherapeutic drugs and deoxycholic acid stimulation in esophageal adenocarcinoma remains to be further explored. This review summarizes current research progress on ROS and stemness activity, regulation of ROS by stanniocalcin-1 (STC1)/uncoupling protein 2 (UCP2), and inspiration for ROS in esophageal adenocarcinoma to guide further research and provide insight into the clinical treatment of esophageal adenocarcinoma.
\end{abstract}

Keywords: Reactive oxygen species (ROS); stanniocalcin-1/uncoupling protein 2 STC1/UCP2; esophageal adenocarcinoma; deoxycholic acid; cancer stem cells

Submitted Sep 25, 2019. Accepted for publication Jan 01, 2021.

doi: $10.21037 /$ tcr-19-1985

View this article at: http://dx.doi.org/10.21037/tcr-19-1985

$\wedge$ ORCID: 0000-0003-2816-9441. 


\section{Introduction}

\section{The origin of reactive oxygen species (ROS)}

ROS are defined as oxygen-containing substances that readily oxidize other molecules and include superoxide $\left(\mathrm{O}_{2}^{-}\right)$, hydroxyl $(\mathrm{HO})$, and hydrogen peroxide $\left(\mathrm{H}_{2} \mathrm{O}_{2}\right)$ molecules (1). ROS are produced in the mitochondria by nicotinamide adenine dinucleotide phosphate (NADPH) oxidase (NOX). G-protein-coupled receptor 5 (TGR5), NOX1, and NOX2 are NADPH hydrogenases. Early studies have shown that acids and bile acids produce ROS by upregulating TGR5 $(2,3)$. Recent studies have shown that bile acids also produce ROS by upregulating NOX1 and NOX2, causing DNA damage and the malignant progression of Barrett's esophagus. Meanwhile, NOX1 and NOX2 inhibition by siRNA interference can reduce ROS production and DNA damage (4). Both P16 and P53 are important tumor suppressor genes. Hong et al. (2) showed that ROS methylate P16DNA and downregulate P16mRNA expression, promote cell proliferation, and cause the malignant transformation of Barrett's esophagus into esophageal adenocarcinoma. Cardin et al. (5) showed that the accumulation of DNA oxidative damage activates telomerase and telomere lengthening occurs in the late stage, ultimately leading to $p 53$ gene mutation and adverse effects on cell growth, apoptosis, and DNA repair that promote cancer progression.

\section{The relationship between ROS and esophageal adenocarcinoma}

ROS do not only cause cancer by damaging DNA, but also by activating inflammatory pathways and producing inflammatory mediators, which further promote tumor microenvironment formation. These processes are related to apoptosis resistance, invasion, and metastasis. Nuclear

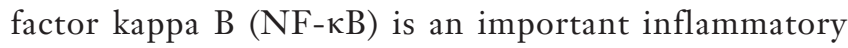
pathway in esophageal adenocarcinoma. Liu et al. (6) found that deoxycholic acid-mediated NF- $\mathrm{BB}$-interleukin 8 (IL-8) pathway activation is related to ROS, and the activated NF- $\mathrm{NB}$ pathway also produces anti-apoptotic protein Bcl-2, rendering DNA-damaged cells resistant to apoptosis (7). Signal transducer and activation of transcription 3 (STAT3) is also an important inflammatory pathway in esophageal adenocarcinoma. Acid and bile acid reflux have been shown to activate the interleukin 6 (IL-6)-STAT3 pathway and increase expression of the antiapoptotic protein Bcl-xL, leading to apoptosis resistance in cancer cells (8). STAT3 inhibitors significantly reduce the proliferation and migration of esophageal adenocarcinoma cells, suggesting that the STAT3 pathway is also associated with esophageal adenocarcinoma proliferation and metastasis (9). Studies have found that ROS activation promote malignant lung cancer progression by stimulating the Janus kinase (JAK)-STAT3 pathway. After UCP2 silencing, ROS levels increase, thus activating the STAT3 pathway. Rotenone or NOX inhibitor pretreatment reduces ROS and eliminates STAT3 pathway activation (10). In addition, studies on gastric cancer have found that $H$. pylori promotes the STAT3 pathway activation by increasing ROS production, further leading to Wnt pathway activation and excessive cancer cell proliferation, while antioxidants significantly inhibit STAT3 pathway activation and prevent malignant cancer progression (11). However, whether ROS can also activate the STAT3 pathway in esophageal adenocarcinoma has not been studied yet.

\section{Reflux of bile acid and ROS in esophageal adenocarcinoma}

It is currently suggested that bile acid reflux is an important factor leading to the formation and progression of Barrett's esophagus and esophageal adenocarcinoma. Bile acids are structurally classified into two groups: free bile acidsincluding cholic acid, deoxycholic acid, goose deoxycholic acid, and a small amount of licholic acid-and the conjugated products of bile acid with glycine or taurine, termed the conjugated bile acid. Bile acid reflux in patients with Barrett's esophagus usually ranges from 3 to $820 \mu \mathrm{M}$, with a median of $180 \mu \mathrm{M}$ (12), but may reach 6,400 $\mu \mathrm{M}$ (13). In the rat reflux model, it was found that gastric acid alone could not induce Barrett's esophagus formation; in contrast, bile acid alone could produce it (14). Shen et al. (15) successfully reprogramed deoxycholic acidinduced immortal esophageal squamous epithelial cell line Het-1a to express caudal-type homeobox gene 2 (CDX2) and mucin 2 (MUC2), which serve as intestinal epithelial cell markers. Huo et al. (7) treated Barrett's esophagus cell lines with deoxycholic acid and ursodeoxycholic acid and found that deoxycholic acid causes oxidative stress reaction and subsequent cell DNA damage, while hydrophobic ursodeoxycholic acid does not damage cell DNA. Esophageal adenocarcinoma is defined as an inflammationrelated cancer in which excessive ROS is mediated by bile acid (deoxycholic acid) reflux (6). Therefore, unconjugated deoxycholic acid may be an important determinant in bile that causes carcinogenesis and the transformation 
of esophageal squamous epithelial cells into Barrett's esophagus (16). Specifically, the molecular mechanism by which deoxycholic acid induces malignant transformation remains unclear.

\section{ROS and cancer stem cells}

Current conventional chemoradiotherapy kills most cancer cells by increasing ROS levels in the cells, but cancer stem cells survive due to their resistance to chemoradiotherapy and thus can induce relapse by differentiating into cancer cells at the appropriate timing. Cancer stem cells are a small number of self-renewing and differentiating cells in tumors, which are closely related to recurrence, metastasis, and chemoradiotherapy resistance. Cancer stem cells may be transformed from normal stem cells, as well as progenitor, tumor, or normal non-stem cells after dedifferentiation and reprogramming (17-19). Therefore, it is particularly important to understand cancer stem cell formation to subsequently perform targeted treatment to improve the efficacy of cancer treatment and improve the survival prognosis of patients.

This review focuses on the research progress of ROS specifically in esophageal adenocarcinoma, including the relationship between stemness activity and ROS, the regulation of ROS, and the inspiration for clinical treatment based on ROS levels.

\section{ROS and stemness activity in esophageal adenocarcinoma}

\section{Markers of cancer stem cells in esophageal adenocarcinoma}

The critical developmental stage of esophageal adenocarcinoma involves characteristic cellular morphological changes, which may suggest cancer stem cell involvement. Esophageal adenocarcinoma developed from the normal esophagus proceeds through several developmental stages, including Barrett's esophagus, low-grade dysplasia, high-grade dysplasia, and esophageal adenocarcinoma. The critical precancerous stage Barrett's esophagus has been marked by columnar epithelial cells replacing squamous epithelial cells in the distal esophagus (20), which strongly suggests the involvement of stem cell-related activities (21).

Three types of markers can be used for the recognition of cancer stem cells, including cell surface molecules, transcription factors, and molecules of signaling pathways. Cell surface molecules mainly include aldehyde dehydrogenase (ALDH), CD44, and CD133, while transcription factors include Octamer-binding transcription factor 4 (OCT4), homeobox protein (Nanog), and sexdetermining region Y-box 2 (SOX2). The signaling pathways include Notch, JAK/STAT3, phosphatidylinositol 3-kinase/ protein kinase (PIK3/AKT), Wnt, etc. (19). In esophageal adenocarcinoma, researchers have found that cancer stem cells of esophageal adenocarcinoma express stem cell markers, such as ALDH (22), OCT4 (23), CD44 (21), and CD133 (21). Lynam-Lennon et al. (22) confirmed that ALDH expression was associated with cancer stem cells. Patients with esophageal adenocarcinoma and elevated ALDH expression were less responsive to chemoradiotherapy. In addition, studies have found that ALDH1+ cells, which can prevent ROS-induced apoptosis by reducing ROS production, are more resistant to oxidative stress than ALDH1 - cells (24). ALDH expression inhibition target cancer stem cells in ovarian cancer, enhancing the sensitivity of ovarian cancer to chemotherapy from taxanes and platinum. These results suggest that cancer stem cells in esophageal adenocarcinoma may also develop resistance to radiotherapy and chemotherapy through ALDH expression to improve antioxidant capacity in cancer. OCT4, also known as OCT3, is an important transcription factor that maintains the pluripotency of stem cells. Studies have shown that OCT4 expression increases with the progression of Barrett's esophagus to esophageal adenocarcinoma, while the decrease in OCT4 expression reduces the invasiveness and cloning ability in esophageal adenocarcinoma cells, reducing cancer stem cell generation. This suggests that OCT4 might play an important role in the progression of Barrett's esophagus to esophageal adenocarcinoma by promoting cancer stem cell formation (23). Signaling pathways such as Notch have been proven to drive the formation of esophageal adenocarcinoma cancer stem cells, while inhibition of Notch signaling could reduce the number of cancer stem cells, increasing their sensitivity to chemoradiotherapeutic drugs (25). The JAK/STAT3 pathway is also recognized as an important signaling pathway regulating the formation of cancer stem cells. It has been found in lung cancer that STAT3 could regulate ALDH activity, which is essential for the maintenance of lung cancer stem cells $(26,27)$. In cervical cancer, STAT3 affects the expression of OCT4 and Nanog transcription factors and regulates the biological characteristics of cervical cancer stem cells (28). It has also been found in esophageal adenocarcinoma that the JAK/STAT3 pathway is activated under the stimulation of 
acid or deoxycholic acid to promote apoptosis resistance, proliferation, and metastasis $(8,9)$. However, whether the STAT3 pathway in esophageal adenocarcinoma can regulate stem cell formation remains unclear, and further studies are needed.

\section{ROS induces stemness activity in cancer}

Studies have shown that low and medium ROS concentrations promote stemness formation in cancer cells (29). In fact, it is one of the reasons for cancer resistance to chemoradiotherapy and its recurrence after chemoradiotherapy. Chemoradiotherapy kills cancer cells by producing ROS; although high ROS concentrations kill cancer cells, playing a therapeutic role, low ROS concentrations act as signaling molecules in related signaling pathways, inducing cancer cells to transform into cancer stem cells and enhancing cancer malignancy $(30,31)$. Both insufficient chemotherapeutic drugs or antioxidant system enhancement in cancer might have the opposite consequence to the desired therapeutic outcome.

ROS can directly activate Notch, STAT3, and other signaling pathways to induce cancer stem cell formation. Charles et al. (32) found that nitric oxide activates Notch signaling and promotes the stemness of glioma cells. Zhang et al. (29) found that during the treatment of pancreatic cancer, low-dose gemcitabine also induces pancreatic cancer stem cell formation by activating the STAT3 pathway through producing low and medium levels of ROS. However, the activated Notch or STAT3 signaling pathway upregulates ROS scavenger enzymes or increases anaerobic glycolysis through the phosphatidylinositol 3 kinase/protein kinase B (PIK3/AKT) pathway to control ROS levels (33), which helps to maintain a low level in cancer stem cells. In addition, transcription factors such as hypoxia-induced factor $\alpha(\mathrm{HIF}-\alpha)$ and NF- $\mathrm{kB}$ also promote the formation or maintenance of stemness in cancer. Researchers have found that ROS promotes cancer stem cell formation by regulating $\mathrm{HIF}-\alpha$ expression and promoting NF- $\kappa \mathrm{B}$ activation (34). Notably, ROS also induce mesenchymal transformation in tumor epithelial cells, promoting invasion and metastasis. As an E-cadherin inhibitor, Snail is the most important mesenchymal transformation transcriptional regulator. Studies have shown that ROS induce Snail expression, which could further activate Notch, PIK3/AKT, Wnt, and other signaling pathways to promote mesenchymal transformation in cancer (19). Mesenchymal transformation is closely related to cancer stem cell formation $(35,36)$, possibly because mesenchymal transformation and cancer stem cells share common activation pathways, such as Notch, PIK3/ AKT, Wnt, and others. Furthermore, it was found that mesenchymal transformation also activates cancer cells to obtain stemness through the Ras-mitogen-activated protein kinase pathway (37). In general, ROS induce cancer stem cell formation by directly activating the relevant signaling pathways or transcription factors of cancer stem cells, and also promote cancer stem cell generation by promoting the occurrence of cancer mesenchymal transformation. However, in esophageal adenocarcinoma, the relationship between ROS and cancer stem cells is still not clear. Studies have found that chemotherapeutic drugs also induce the formation of esophageal adenocarcinoma cancer stem cells (38). Bile acid stimulation upregulates esophageal adenocarcinoma stem cell markers and promotes cancer stem cell formation (23). It is known that both chemotherapeutic drug use and deoxycholic acid stimulation promote ROS generation. However, whether the induction of cancer stem cell formation by chemotherapeutic drugs and deoxycholic acid stimulation in esophageal adenocarcinoma is related to ROS remains to be further explored.

\section{ROS uncoupling protein 2 (UCP2) and stanniocalcin-1 (STC1)}

Proton leak, a significant biological energy phenomenon that greatly reduces the yield of ROS and adenosine triphosphate (ATP), is one of the important accomplices of drug resistance in cancer cells. Significantly high proton leak-mediated bioelectricity is one of the primary characteristics of cancer, rendering cancer cells different from normal cells $(39,40)$. Although proton leak has not been directly studied in relation to cancer treatment, it plays a key role in cancer treatment strategies because proton leak determines the level of ROS. The up-regulated UCP2 expression in the UCP2-mediated proton leak in cancer cells is responsible for drug resistance. Reducing UCP2-mediated proton leak increased the level of ROS and chemical sensitivity to cisplatin therapy in cancer cells (40).

Studies have found that deoxycholic acid could reduce the expression of UCP2 and enhance the ability of cloning, proliferation, invasion, and metastasis in esophageal adenocarcinoma cells (41). UCP2 was found to be widely distributed in the liver, brain, pancreas, adipose tissue, immune cells, spleen, kidney, and central nervous system. Several studies have focused on UCP2 (42), which can pump protons between the inner and outer mitochondrial 
membranes into the mitochondrial matrix. Proton transport bypasses ATP synthase and reduces the proton gradient of the mitochondrial inner membrane, leaving the oxidative complex of the electron transport chain in the mitochondrial inner membrane idle. This leads to a decrease in the mitochondrial inner membrane potential, electron leakage in the electron transport chain, and ROS production. Functionally, UCP2 is involved in insulin secretion, immune response regulation, and cell energy metabolism, which enhance glycolysis (43).

In recent years, studies have identified a key role of UCP2 in oncogenesis and progression. It is generally believed that UCP2 is inhibited in the early stage of carcinogenesis, during which intracellular ROS accumulation leads to genomic instability and induces cancer formation. In the subsequent stages of cancer development, UCP2 is activated or overexpressed in cancer cells compared to in normal tissue cells by reducing intracellular ROS levels, thereby increasing resistance to chemotherapeutic drugs in cancer cells, improving their invasiveness, and protecting cancer cells $(42,44,45)$. Therefore, UCP2 and cancer might have a dual relationship. Proton leak determines the ROS level, which plays an important role in cancer treatment strategies. Reduction of mitochondrial oxidative metabolism is a hallmark biological energy feature of malignant tumors, and may have an adaptive effect on cancer occurrence. Targeting mitochondria is a promising new approach for cancer prevention and treatment (20). UCP2 is often overexpressed in drug-resistant cancer cells, where it controls the ROS level and limits drug toxicity.

STC1 is a blood calcium and phosphorus metabolic balancing regulating protein expressed in various tissues and organs in the human body. In addition, it is involved in calcium and phosphorus transport and plays an important role in the formation and progression of cancer. Studies have shown that STC1 is overexpressed in multiple malignant tumors, such as laryngeal cancer, glioma, esophageal cancer, gastric cancer, colorectal cancer, thyroid cancer, kidney cancer, and breast cancer. STC1 promotes cell proliferation by regulating cell cycle protein expression (46). In kidney cancer, STC1 accelerates transformation from G1 phase to $\mathrm{S}$ phase, thereby shortening the cell cycle and increasing the proliferation rate of cancer cells (47). STC1 improves the anti-apoptosis effect in cancer, mainly by activating the ERK and JNK signaling pathways, promoting the expression of antiapoptotic proteins $\mathrm{Bcl}-2$ and $\mathrm{Bcl}-\mathrm{xL}$, and inhibiting the pro-apoptotic proteins Bax, Bak, and Bid (48). Moreover,
STC1 also promotes blood vessel formation through the vascular endothelial growth factor/vascular endothelial growth factor receptor (VEGF/VEGFR2)-related signaling pathway (49). Therefore, the high expression of STC1 improves the malignant ability of the cancer and is a predictive marker of cancer progression. Wang et al. (50) showed that STC1 induces UCP2 expression in macrophages, which is an important factor that weakens the oxidative stress response in cells. Ono et al. (51) reported that large STC1 quantity mediates UCP2, reduces alveolar epithelial cell ROS production, and inhibits pulmonary fibrosis. These findings verify the existence of an association between STC1 and UCP2 in normal cells. Recent studies have found that STC1 is highly expressed in cancer, accompanied by a decrease in intracellular ROS (52). Considering the involvement of UCP2 in regulating cellular ROS production, it is speculated that the reduction of STC1 to ROS depends on the expression of $\mathrm{UCP} 2$ in related cancers.

\section{ROS on the prevention and treatment of esophageal adenocarcinoma}

\section{Prevention of cancer (ROS lowering anticancer therapy)}

It is known that deoxycholic acid stimulates the production of ROS, and the increased ROS triggers a series of malignant events. ROS can promote HIF-2a generation (53) and NF- $\mathrm{\kappa B}$ activation (54) by damaging DNA (55) and interfering with the expression of tumor suppressor genes $(2,5,56)$, thus inducing anticancer immune tolerance and promoting the expression of antiapoptotic proteins (53), which together promote esophageal adenocarcinoma occurrence. On one hand, oxidative stress plays an important role in esophageal adenocarcinoma occurrence; therefore, antioxidant therapy may be effective in the prevention and treatment of esophageal adenocarcinoma, specifically by inhibiting ROS production and increasing ROS clearance. For example, NADPH oxidase is the key enzyme for peroxisomal ROS production. Studies have shown that inhibiting NADPH oxidase ROS species production and DNA damage, which may be an effective target for preventing tumorigenesis (4). On the other hand, an obstacle to the antioxidant system leads to an increase in ROS due to limited clearance. Studies have found decreased levels of superoxide dismutase (SOD) in Barrett's esophagus and esophageal adenocarcinoma, where SOD supplementation reduces oxidative damage to 
the esophageal epithelium and prevents the progression of esophageal epithelium from Barrett's esophagus to esophageal adenocarcinoma (57). In addition, some antioxidants may be effective in the prevention of esophageal adenocarcinoma. Bhardwaj et al. (58) found that a natural antioxidant, oleandine, which may be used to prevent the occurrence of esophageal adenocarcinoma, inhibits ROS production, prevents DNA damage in Barrett's esophagus cells, and enhances DNA repair. Diallyl disulfide is a natural organic sulfur compound derived from garlic. Studies have shown that diallyl disulfide, which may be a good candidate for Barrett's esophagus or esophageal adenocarcinoma chemoprophylactic and therapeutic therapies, inhibits deoxycholic acid-induced ROS production, thereby inhibiting NF- $\kappa \mathrm{B}$ activation and inflammatory cytokine and anti-apoptotic protein production (59). In addition, Jenkins et al. (60) confirmed that vitamin $\mathrm{C}$ blocks the genetic toxicity of esophageal mucosal epithelium caused by bile acids by clearing ROS, thus supporting the idea that antioxidants may be an effective chemical protectant. In short, ROS promotes the occurrence of esophageal adenocarcinoma through a variety of mechanisms. Therefore, lowering the ROS level in the body might contribute to the prevention of esophageal adenocarcinoma. Specifically, these measures could be achieved by inhibiting key enzymes involved in ROS production, enhancing the body antioxidant system, or using some antioxidants to reduce ROS levels in the body. However, effectiveness still needs to be confirmed using sufficient evidence. It is worth noting that antioxidant therapy may be effective for the prevention of cancer, but for malignant developed cancer, especially advanced cancer, antioxidant therapy is not helpful and may reduce the survival rate of tumor patients. This may be due to the elimination of ROS as well as the ROS-mediated apoptosis, leading to the survival of cancer cells. Therefore, it is necessary to increase the level of ROS to induce apoptosis in an advanced situation.

\section{Cancer therapy (an anticancer therapy that increases ROS)}

There are still no strategies for the treatment of esophageal adenocarcinoma. At present, neoadjuvant chemoradiotherapy combined with surgery is utilized, but the response rate of tumor to chemoradiotherapy is $16 \%$ of the complete pathological responses (34). Researchers have found that the existence of esophageal adenocarcinoma cancer stem cell subsets is important for resistance to neoadjuvant chemoradiotherapy; therefore, targeted treatment of cancer stem cells may increase the sensitivity of chemoradiotherapy and improve clinical results (25). It is known that the concentration of ROS in cancer stem cells is also lower than that in ordinary cancer cells, because cancer stem cells are more sensitive to ROS. Medium and low concentrations of ROS can help the formation of cancer stem cells, while high concentrations can inhibit the expression of cancer stem cells and even kill them. Therefore, current chemoradiotherapeutic drugs kill cancer and cancer stem cells by producing a large amount of ROS. However, cancer stem cells can reduce the concentration of ROS in cells through a powerful antioxidant system or the transformation of energy metabolism. In terms of antioxidant systems, cancer stem cells can enhance antioxidant capacity by inducing the activation of antioxidant protein nuclear factor erythroid 2-related factor 2 (NRF2) (61), increasing the expression of antioxidant enzyme superoxide dismutase 1 (62), and increasing the production of antioxidant glutathione (63), to control the ROS level and avoid damage to cancer cells. Therefore, ROS levels may be low in this case, which may induce the formation of cancer stem cells, which is also a cause of cancer chemoradiotherapy resistance. Thus, under the condition of ensuring sufficient ROS production by chemotherapy, drugs that inhibit cancer antioxidant system can be combined to significantly increase ROS levels in cancer to achieve therapeutic purpose. Studies have shown that a combination of cisplatin and salazosulfapyridine can reduce the in vivo glutathione effect, leading to significant ROS accumulation, thereby enhancing the sensitivity of colorectal cancer to cisplatin and inhibiting cancer cell proliferation (64). Another study found that reducing the antioxidant enzyme $\mathrm{Mn}$-superoxide dismutase (Mn-SOD) expression through siRNA can increase the sensitivity of ovarian cancer cells to chemically induced apoptosis (65). In addition, ALDH expression also contributes to the clearance of ROS in cancer stem cells. High ALDH expression is related to chemotherapeutic cancer resistance, while siRNA ALDH enhances the cytotoxicity of taxane and platinum and targets ovarian cancer stem cells (66). In short, improving the ROS level in cancer may be effective for cancer treatment. Due to the enhanced antioxidant capacity of cancer stem cells, drugs used solely to promote ROS production may be ineffective. Therefore, ROS-generating drugs combined with antioxidant system drugs could effectively improve the ROS level in cancer cells to achieve therapeutic effects. However, at present, few studies have focused on these methods in 
esophageal adenocarcinoma, which still need to be further explored.

\section{Conclusions}

Esophageal adenocarcinoma is an inflammatory cancer caused by the chronic reflux of gastric and bile acids. ROS not only causes cancer by damaging DNA, but also activates inflammatory pathways, produces inflammatory mediators, and further promotes cancer microenvironment formation. ROS levels are also related to apoptosis resistance, invasion, and metastasis, as well as potentially cancer stem cells, and thus may be a key driver of the growth and metastasis of this cancer type to initiate tumorigenesis, self-renewal, and differentiation of cancer cells. In addition, interactions among ROS, UCP2, and STC1 are noteworthy. It is important to recognize that esophageal adenocarcinoma can be prevented or treated by regulating the ROS level in the body. Since high ROS levels can kill cancer cells significantly, the combination of chemotherapeutic drugs along with drugs that exhibit antioxidant system characteristics might serve as an effective treatment strategy.

\section{Acknowledgments}

Funding: None.

\section{Footnote}

Conflicts of Interest: All authors have completed the ICMJE uniform disclosure form (available at http://dx.doi. org/10.21037/tcr-19-1985). The authors have no conflicts of interest to declare.

Ethical Statement: The authors are accountable for all aspects of the work in ensuring that questions related to the accuracy or integrity of any part of the work are appropriately investigated and resolved.

Open Access Statement: This is an Open Access article distributed in accordance with the Creative Commons Attribution-NonCommercial-NoDerivs 4.0 International License (CC BY-NC-ND 4.0), which permits the noncommercial replication and distribution of the article with the strict proviso that no changes or edits are made and the original work is properly cited (including links to both the formal publication through the relevant DOI and the license). See: https://creativecommons.org/licenses/by-nc-nd/4.0/.

\section{References}

1. Larosa $V$, Remacle C. Insights into the respiratory chain and oxidative stress. Biosci Rep 2018;38:BSR20171492.

2. Hong J, Resnick M, Behar J, et al. Acid-induced p16 hypermethylation contributes to development of esophageal adenocarcinoma via activation of NADPH oxidase NOX5-S. Am J Physiol Gastrointest Liver Physiol 2010;299:G697-706.

3. Li D, Cao W. Bile acid receptor TGR5, NADPH oxidase NOX5-S and CREB mediate bile acid-induced DNA damage in Barrett's esophageal adenocarcinoma cells. Sci Rep 2016;6:31538.

4. Bhardwaj V, Gokulan RC, Horvat A, et al. Activation of NADPH oxidases leads to DNA damage in esophageal cells. Sci Rep 2017;7:9956.

5. Cardin R, Piciocchi M, Tieppo C, et al. Oxidative DNA damage in Barrett mucosa: correlation with telomeric dysfunction and p53 mutation. Ann Surg Oncol 2013;20 Suppl 3:S583-9.

6. Liu D, Zhang R, Wu J, et al. Interleukin-17A promotes esophageal adenocarcinoma cell invasiveness through ROS-dependent, NF-kB-mediated MMP-2/9 activation. Oncol Rep 2017;37:1779-85.

7. Huo X, Juergens S, Zhang X, et al. Deoxycholic acid causes DNA damage while inducing apoptotic resistance through NF- $\mathrm{KB}$ activation in benign Barrett's epithelial cells. Am J Physiol Gastrointest Liver Physiol 2011;301:G278-86.

8. Dvorak K, Chavarria M, Payne CM, et al. Activation of the interleukin-6/STAT3 antiapoptotic pathway in esophageal cells by bile acids and low $\mathrm{pH}$ : relevance to Barrett's esophagus. Clin Cancer Res 2007;13:5305-13.

9. Timme S, Ihde S, Fichter CD, et al. STAT3 expression, activity and functional consequences of STAT3 inhibition in esophageal squamous cell carcinomas and Barrett's adenocarcinomas. Oncogene 2014;33:3256-66.

10. Li W, Nichols K, Nathan CA, et al. Mitochondrial uncoupling protein 2 is up-regulated in human head and neck, skin, pancreatic, and prostate tumors. Cancer Biomark 2013;13:377-83.

11. Park B, Lim JW, Kim H. Lycopene treatment inhibits activation of Jak1/Stat 3 and $\mathrm{Wnt} / \beta$-catenin signaling and attenuates hyperproliferation in gastric epithelial cells. Nutr Res 2019;70:70-81.

12. Nehra D, Howell P, Williams CP, et al. Toxic bile acids in gastro-oesophageal reflux disease: influence of gastric acidity. Gut 1999;44:598-602. 
13. Iftikhar SY, Ledingham S, Steele RJ, et al. Bile reflux in columnar-lined Barrett's oesophagus. Ann R Coll Surg Engl 1993;75:411-6.

14. Sun D, Wang X, Gai Z, et al. Bile acids but not acidic acids induce Barrett's esophagus. Int J Clin Exp Pathol 2015;8:1384-92.

15. Shen C, Zhang H, Wang P, et al. Deoxycholic acid (DCA) confers an intestinal phenotype on esophageal squamous epithelium via induction of the stemness-associated reprogramming factors OCT4 and SOX2. Cell Cycle 2016;15:1439-49.

16. Wang KK, Sampliner RE. Updated guidelines 2008 for the diagnosis, surveillance and therapy of Barrett's esophagus. Am J Gastroenterol 2008;103:788-97.

17. Teng YD, Wang L, Kabatas S, et al. Cancer stem cells or tumor survival cells? Stem Cells Dev 2018;27:1466-78.

18. Cabrera MC, Hollingsworth RE, Hurt EM. Cancer stem cell plasticity and tumor hierarchy. World J Stem Cells 2015;7:27-36.

19. Lee SY, Jeong EK, Ju MK, et al. Induction of metastasis, cancer stem cell phenotype, and oncogenic metabolism in cancer cells by ionizing radiation. Mol Cancer 2017;16:10.

20. Donadelli M, Dando I, Dalla PE, et al. Mitochondrial uncoupling protein 2 and pancreatic cancer: a new potential target therapy. World J Gastroenterol 2015;21:3232-8.

21. Mokrowiecka A, Veits L, Falkeis C, et al. Expression profiles of cancer stem cell markers: CD133, CD44, Musashi-1 and EpCAM in the cardiac mucosa-Barrett's esophagus-early esophageal adenocarcinoma-advanced esophageal adenocarcinoma sequence. Pathol Res Pract 2017;213:205-9.

22. Lynam-Lennon N, Heavey S, Sommerville G, et al. MicroRNA-17 is downregulated in esophageal adenocarcinoma cancer stem-like cells and promotes a radioresistant phenotype. Oncotarget 2017;8:11400-13.

23. Wang X, Yang S, Zhao X, et al. OCT3 and SOX2 promote the transformation of Barrett's esophagus to adenocarcinoma by regulating the formation of tumor stem cells. Oncol Rep 2014;31:1745-53.

24. Vishnubalaji R, Manikandan M, Fahad M, et al. Molecular profiling of ALDH1(+) colorectal cancer stem cells reveals preferential activation of MAPK, FAK, and oxidative stress pro-survival signalling pathways. Oncotarget 2018;9:13551-64.

25. Wang Z, Da ST, Jin K, et al. Notch signaling drives stemness and tumorigenicity of esophageal adenocarcinoma. Cancer Res 2014;74:6364-74.
26. Codony-Servat J, Codony-Servat C, Cardona AF, et al. Cancer stem cell biomarkers in EGFR-mutationpositive non-small-cell lung cancer. Clin Lung Cancer 2019;20:167-77.

27. Shao C, Sullivan JP, Girard L, et al. Essential role of aldehyde dehydrogenase $1 \mathrm{~A} 3$ for the maintenance of nonsmall cell lung cancer stem cells is associated with the STAT3 pathway. Clin Cancer Res 2014;20:4154-66.

28. Wang H, Deng J, Ren HY, et al. STAT3 influences the characteristics of stem cells in cervical carcinoma. Oncol Lett 2017;14:2131-6.

29. Zhang Z, Duan Q, Zhao H, et al. Gemcitabine treatment promotes pancreatic cancer stemness through the Nox/ ROS/NF- $\mathrm{B} / \mathrm{STAT} 3$ signaling cascade. Cancer Lett 2016;382:53-63.

30. Qian X, Nie X, Yao W, et al. Reactive oxygen species in cancer stem cells of head and neck squamous cancer. Semin Cancer Biol 2018;53:248-57.

31. Liao Z, Chua D, Tan NS. Reactive oxygen species: a volatile driver of field cancerization and metastasis. Mol Cancer 2019;18:65.

32. Charles N, Ozawa T, Squatrito M, et al. Perivascular nitric oxide activates notch signaling and promotes stem-like character in PDGF-induced glioma cells. Cell Stem Cell 2010;6:141-52.

33. Ding S, Li C, Cheng $\mathrm{N}$, et al. Redox regulation in cancer stem cells. Oxid Med Cell Longev 2015;2015:750798.

34. Wozny AS, Lauret A, Battiston-Montagne $\mathrm{P}$, et al. Differential pattern of HIF- $1 \alpha$ expression in HNSCC cancer stem cells after carbon ion or photon irradiation: one molecular explanation of the oxygen effect. Br J Cancer 2017;116:1340-49.

35. Jing L, Ruan Z, Sun H, et al. Epithelial-mesenchymal transition induced cancer-stem-cell-like characteristics in hepatocellular carcinoma. J Cell Physiol 2019;234:18448-58.

36. Wang R, Sun Q, Wang P, et al. Notch and Wnt/ $\beta$-catenin signaling pathway play important roles in activating liver cancer stem cells. Oncotarget 2016;7:5754-68.

37. Morel AP, Lièvre M, Thomas $C$, et al. Generation of breast cancer stem cells through epithelial-mesenchymal transition. PLoS One 2008;3:e2888.

38. Sun X, Martin R, Zheng Q, et al. Drug-induced expression of EpCAM contributes to therapy resistance in esophageal adenocarcinoma. Cell Oncol (Dordr) 2018;41:651-62.

39. Wang R, MoYung KC, Zhang MH, et al. UCP2- and non-UCP2-mediated electric current in eukaryotic cells exhibits different properties. Environ Sci Pollut Res Int 
2015;22:19618-31.

40. Wang R, MoYung KC, Zhao YJ, et al. A mechanism for the temporal potentiation of genipin to the cytotoxicity of cisplatin in colon cancer cells. Int J Med Sci 2016;13:507-16.

41. Xu Y, Feingold PL, Surman DR, et al. Bile acid and cigarette smoke enhance the aggressive phenotype of esophageal adenocarcinoma cells by downregulation of the mitochondrial uncoupling protein-2. Oncotarget 2017;8:101057-71.

42. Robbins D, Zhao Y. New aspects of mitochondrial uncoupling proteins (UCPs) and their roles in tumorigenesis. Int J Mol Sci 2011;12:5285-93.

43. Kuai XY, Ji ZY, Zhang HJ. Mitochondrial uncoupling protein 2 expression in colon cancer and its clinical significance. World J Gastroenterol 2010;16:5773-78.

44. Pitt MA. Overexpression of uncoupling protein-2 in cancer: metabolic and heat changes, inhibition and effects on drug resistance. Inflammopharmacology 2015;23:365-9.

45. Yu J, Shi L, Lin W, et al. UCP2 promotes proliferation and chemoresistance through regulating the $\mathrm{NF}-\kappa \mathrm{B} /$ $\beta$-catenin axis and mitochondrial ROS in gallbladder cancer. Biochem Pharmacol 2020;172:113745.

46. Liu G, Yang G, Chang B, et al. Stanniocalcin 1 and ovarian tumorigenesis. J Natl Cancer Inst 2010;102:812-27.

47. Ma X, Gu L, Li H, et al. Hypoxia-induced overexpression of stanniocalcin-1 is associated with the metastasis of early stage clear cell renal cell carcinoma. J Transl Med 2015;13:56.

48. Du YZ, Gu XH, Cheng SF, et al. The oncogenetic role of stanniocalcin 1 in lung adenocarcinoma: a promising serum candidate biomarker for tracking lung adenocarcinoma progression. Tumour Biol 2016;37:5633-44.

49. Higuera GA, Fernandes H, Spitters TW, et al. Spatiotemporal proliferation of human stromal cells adjusts to nutrient availability and leads to stanniocalcin-1 expression in vitro and in vivo. Biomaterials 2015;61:190-202.

50. Wang Y, Huang L, Abdelrahim M, et al. Stanniocalcin-1 suppresses superoxide generation in macrophages through induction of mitochondrial UCP2. J Leukoc Biol 2009;86:981-8.

51. Ono M, Ohkouchi S, Kanehira M, et al. Mesenchymal stem cells correct inappropriate epithelial-mesenchyme relation in pulmonary fibrosis using stanniocalcin-1. Mol Ther 2015;23:549-60.

52. Ohkouchi S, Block GJ, Katsha AM, et al. Mesenchymal stromal cells protect cancer cells from ROS-induced apoptosis and enhance the Warburg effect by secreting STC1. Mol Ther 2012;20:417-23.

53. Huo X, Agoston AT, Dunbar KB, et al. Hypoxia-inducible factor- $2 \alpha$ plays a role in mediating oesophagitis in GORD. Gut 2017;66:1542-54.

54. Jenkins GJ, Cronin J, Alhamdani A, et al. The bile acid deoxycholic acid has a non-linear dose response for DNA damage and possibly NF-kappaB activation in oesophageal cells, with a mechanism of action involving ROS. Mutagenesis 2008;23:399-405.

55. Thanan R, Ma N, Hiraku Y, et al. DNA damage in CD133-positive cells in Barrett's esophagus and esophageal adenocarcinoma. Mediators Inflamm 2016;2016:7937814.

56. Ding N, Maiuri AR, O Hagan HM. The emerging role of epigenetic modifiers in repair of DNA damage associated with chronic inflammatory diseases. Mutation Research/ Reviews in Mutation Research 2019;780:69-81.

57. Martin RC, Liu Q, Wo JM, et al. Chemoprevention of carcinogenic progression to esophageal adenocarcinoma by the manganese superoxide dismutase supplementation. Clin Cancer Res 2007;13:5176-82.

58. Bhardwaj V, Horvat A, Korolkova O, et al. Prevention of DNA damage in Barrett's esophageal cells exposed to acidic bile salts. Carcinogenesis 2016;37:1161-9.

59. Feng C, Luo Y, Nian Y, et al. Diallyl disulfide suppresses the inflammation and apoptosis resistance induced by DCA through ROS and the NF- $\mathrm{KB}$ signaling pathway in human Barrett's epithelial cells. Inflammation 2017;40:818-31.

60. Jenkins GJ, D'Souza FR, Suzen SH, et al. Deoxycholic acid at neutral and acid $\mathrm{pH}$, is genotoxic to oesophageal cells through the induction of ROS: The potential role of anti-oxidants in Barrett's oesophagus. Carcinogenesis 2007;28:136-42.

61. Ryoo IG, Choi BH, Ku SK, et al. High CD44 expression mediates p62-associated NFE2L2/NRF2 activation in breast cancer stem cell-like cells: Implications for cancer stem cell resistance. Redox Biol 2018;17:246-58.

62. Glasauer A, Chandel NS. Targeting antioxidants for cancer therapy. Biochem Pharmacol 2014;92:90-101.

63. Peng G, Tang Z, Xiang Y, et al. Glutathione peroxidase 4 maintains a stemness phenotype, oxidative homeostasis and regulates biological processes in Panc-1 cancer stem-like cells. Oncol Rep 2019;41:1264-74.

64. Ma MZ, Chen G, Wang P, et al. Xc- inhibitor sulfasalazine sensitizes colorectal cancer to cisplatin by a GSH- 
dependent mechanism. Cancer Lett 2015;368:88-96.

65. Yeung BH, Wong KY, Lin MC, et al. Chemosensitisation by manganese superoxide dismutase inhibition is caspase-9 dependent and involves extracellular signal-regulated

Cite this article as: $\mathrm{Hu}$ Y, Ye X, Wang R, Poon K. Current research progress in the role of reactive oxygen species in esophageal adenocarcinoma. Transl Cancer Res 2021;10(3):15681577. doi: $10.21037 /$ tcr-19-1985 kinase 1/2. Br J Cancer 2008;99:283-93.

66. Landen CN Jr, Goodman B, Katre AA, et al. Targeting aldehyde dehydrogenase cancer stem cells in ovarian cancer. Mol Cancer Ther 2010;9:3186-99. 